\title{
REVIEW
}

\section{Next-Generation Proteomics and Its Application to Clinical Breast Cancer Research}

\author{
Mariya Mardamshina and Tamar Geiger
}

From the Department of Human Molecular Genetics and Biochemistry, Sackler Faculty of Medicine, Tel Aviv University, Tel Aviv, Israel

\author{
Accepted for publication \\ July 6, 2017. \\ Address correspondence to \\ Tamar Geiger, Ph.D., Sackler \\ Faculty of Medicine, Tel Aviv \\ University, Haim Levanon St. \\ 55, Tel Aviv, Israel. E-mail: \\ geiger@tauex.tau.ac.il.
}

\begin{abstract}
Proteomics technology aims to map the protein landscapes of biological samples, and it can be applied to a variety of samples, including cells, tissues, and body fluids. Because the proteins are the main functional molecules in the cells, their levels reflect much more accurately the cellular phenotype and the regulatory processes within them than gene levels, mutations, and even mRNA levels. With the advancement in the technology, it is possible now to obtain comprehensive views of the biological systems and to study large patient cohorts in a streamlined manner. In this review we discuss the technological advancements in mass spectrometry-based proteomics, which allow analysis of breast cancer tissue samples, leading to the first large-scale breast cancer proteomics studies. Furthermore, we discuss the technological developments in blood-based biomarker discovery, which provide the basis for future development of assays for routine clinical use. Although these are only the first steps in implementation of proteomics into the clinic, extensive collaborative work between these worlds will undoubtedly lead to major discoveries and advances in clinical practice. (Am J Pathol 2017, 187: 2175-2184; http://dx.doi.org/10.1016/j.ajpath.2017.07.003)
\end{abstract}

Omics technologies have revolutionized cancer research, through mapping of somatic mutations, gene copy number variations, and profiling gene expression alterations using genomic technologies, primarily next-generation sequencing. Proteomics, which is the focus of this review, in analogy to genomics, aims to profile the entire protein content of a biological sample, including the protein modifications and interactions. ${ }^{1}$ The samples may be cells, tissues, or body fluids and, specifically in cancer clinical research, can include tumor tissues, plasma, urine, or proximal body fluids. The mainstream proteomic approach is termed bottom-up or shotgun proteomics and is based on mass spectrometry (MS) technology. In this approach proteins are extracted from the biological samples, followed by their digestion to peptides, chromatographic separation, and MS analysis (Figure 1). ${ }^{2}$ Typically, proteins are digested with trypsin, which cleaves after every lysine and arginine residues in the protein, resulting in peptides of approximately 6 to 25 amino acids, which are normally unique to the protein. The MS determines the mass-to-charge ratio of each peptide in the complex mixture of hundreds of thousands of peptides and further fragments each peptide to allow determination of the amino acid sequence. To add a quantitative dimension to the MS analysis, sample preparation is often combined with labeling with stable isotopes. Metabolic labeling with heavy amino acids, known as the stable isotope labeling with amino acids in cell culture (SILAC) technique, or chemical labeling with isobaric tags techniques, such as isobaric tag for relative and absolute quantitation (iTRAQ) or tandem mass tag (TMT), are extensively used for relative quantification of a large variety of sample types. ${ }^{3}$ Combination with heavy proteins or peptides can further provide the absolute amount of selected proteins of interest. All of these data are then computationally analyzed against protein databases that provide the identity (amino acid sequence) and quantitative information

Supported by the European Council ERC grant 639534 PROTEOMICAN (M.M. and T.G).

Disclosures: None declared.

This article is part of a review series on next-generation breast cancer omics. 


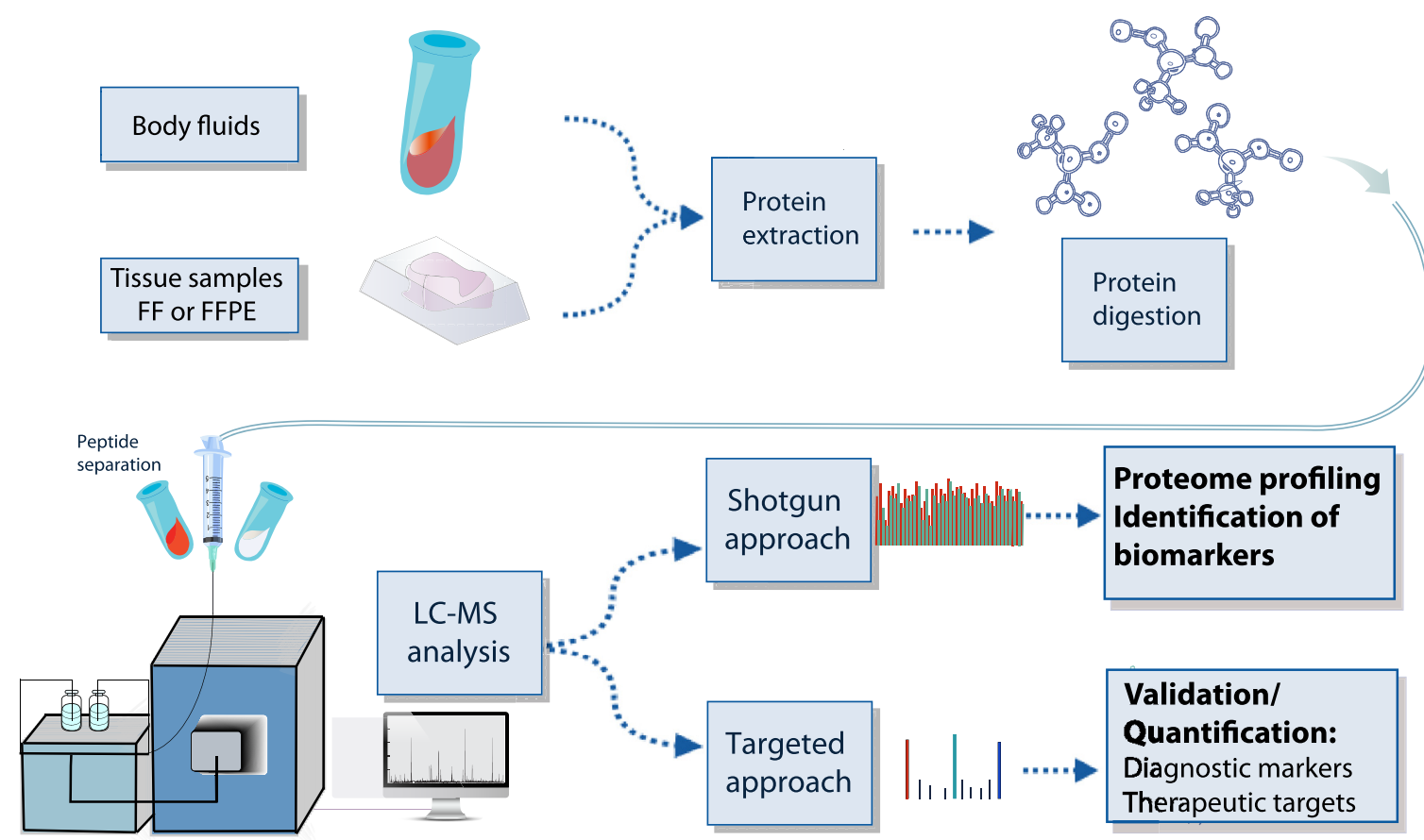

Figure 1 Schematic overview of mass spectrometry (MS)-based workflow used for proteome profiling of tissue samples and biological fluids. MS-based proteomics can be used for identification of novel biomarkers or validation and quantification of specific therapeutic targets in the clinic. Proteins are extracted from various biological samples, digested into peptides, separated by liquid chromatography coupled to MS analysis. FF, fresh frozen; FFPE, formalin-fixed paraffin-embedded; LC, liquid chromatography.

about each peptide and protein. ${ }^{4}$ Dramatic advancements in MS technology in the past decade increased the resolution, mass accuracy, and speed of modern mass spectrometers, thereby allowing higher coverage of the human proteome and increased throughput of the analysis. ${ }^{2}$ As an example, 8 hours of measurement in the MS were required for the identification of approximately 5000 proteins only 5 years ago, whereas recent studies achieved similar results in 90 minutes. ${ }^{5,6}$ Further peptide fractionation before the MS runs enables much more comprehensive coverage of the proteome and increases the identifications to $>10,000$ proteins. $^{7,8}$ This depth allows identification of lowly expressed proteins and therefore opens new possibilities for application of the technology to clinical samples, aiming to reveal disease mechanisms and novel biomarkers.

Beyond the discovery approach, which is based on the genome-scale proteome analysis, MS-based proteomics technology can also be used as a validation approach of specific biomarker candidates and can be applied to routine diagnostics in the clinic (Figure 1). In this targeted MS approach, rather than examining the entire proteome, only predetermined peptides are analyzed with high sensitivity, speed, and quantitative accuracy. ${ }^{9,10}$ In the present review, we focus on the application of the MS technologies to discovery proteomics in breast cancer research and the potential application of targeted proteomics to the clinic. Alternative approaches, such as matrix-assisted laser desorption/ionization (MALDI) imaging or protein-based approaches, which are primarily based on antibodies such as the human protein atlas antibody-based genome-scale initiative or the reverse-phase protein array approach, have been discussed elsewhere ${ }^{11-13}$ and are beyond the scope of this review.

\section{Proteomics versus Genomics Approaches}

Genomic analysis of breast cancer clinical samples has been applied to thousands of tumor samples as a part of the Cancer Genome Atlas ${ }^{14}$ and additional international initiatives. ${ }^{15,16}$ These consortia, aiming to classify breast cancer subtypes and to associate the profiles with cancer prognosis, profiled the gene expression patterns, somatic mutations, copy number variations (CNVs), and epigenetic profiles. Despite this breadth of molecular information, the protein level has been, to a large extent, neglected. To understand the potential contribution of proteomics to the molecular characterization of cancer, multiple studies performed detailed comparisons of proteomic profiles and the genomics and transcriptomics of the same systems. Comparing cancer proteomes with $\mathrm{CNV}$ data showed low correlation of approximately 0.2 , highlighting the relatively minor contribution of these genomic alterations to the levels of their end products, the proteins. ${ }^{17}$ Comparative analysis of breast cancer tissue analysis showed that only $30 \%$ of the chromosomal aberrations are translated to protein abundance changes. ${ }^{18}$ These studies showed that only some of the amplifications and deletions, presumably the cancer 
drivers, have a functional output, whereas most are further controlled by other gene expression regulatory mechanisms of transcription, translation, and mRNA and protein stability. Much higher correlation is observed between the transcriptome and the proteome, because in many systems transcription is the major determinant of protein amount. ${ }^{19,20}$ Cell line studies have shown correlations of 0.4 to 0.6 between mRNA levels and proteins. ${ }^{8,21}$ Although mRNA levels are often used as proxies of protein levels, this limited correlation shows the dramatic differences between these two layers, which reflect the potential contribution and the importance of protein analysis.

Analyzing the protein level has the potential to integrate all of the upstream genetic alterations, epigenetic regulations, and environmental effects that are fundamental regulators of the cancer phenotype. If the technology permitted complete coverage of all full-length proteins, these analyses would identify all expressed mutated proteins, the functional CNVs, and output of all regulatory mechanisms of genes and proteins. However, despite the improved proteomic coverage, it does not yet enable identification of most of the mutated proteins. ${ }^{18}$ Furthermore, to identify mutations by standard proteomic approaches, one would have to have a database, which includes all potentially mutated protein sequences. To obtain such a comprehensive database it is possible to integrate the sequencing information of all somatic mutations obtained in the multitude of genomic databases, such as Catalogue of Somatic Mutations in Cancer and The Cancer Genome Atlas (TCGA). With the growing number of sequenced cancer genomes, several databases, for example CanProVar ${ }^{22,23}$ and XMann, ${ }^{24}$ integrate these data into proteomic databases that include nonsynonymous mutations, including germline and somatic ones. Alternatively, sample-specific databases can be generated directly from genomics of the same samples, when these are analyzed with both technologies. ${ }^{18,25}$ Beyond the identification of mutated proteins, this integrated genomic-proteomic analysis can associate between specific mutations and the proteomic profiles. Specifically in breast cancer, given the moderate mutational load and the high variation in mutational profiles between patients, integration of the genomics with proteomics is capable of revealing the convergence of distinct mutations to a limited number of pathways, which can be identified by proteomics. Moreover, because mutations often occur in regulatory proteins, proteomics can reveal the output of these perturbations and can identify the functional drivers of tumorigenesis. With the use of this approach, Lawrence et $\mathrm{al}^{25}$ analyzed triple-negative breast cancer cell lines and showed that multiple mutations are associated with similar proteomic alteration, thereby reducing cancer heterogeneity on the protein level. These common altered proteins can then be targeted with the same drugs despite their distinct genetic profiles. The Clinical Proteomic Tumor Analysis Consortium performed proteomic and phosphoproteomic analysis of 77 breast cancer tissue samples from the TCGA and could therefore integrate the proteomic data with the genomic data of the same samples (detailed below). With the use of the sample-specific databases, they showed the association of mutations with changes in protein levels. The phosphoproteomic analysis of these tissues further identified signaling pathways that change in association with specific mutations. ${ }^{18}$ For example, they associated between phosphorylation of RPS6KA5 and EIF2AK4 with mutations in PIK3CA, and phosphorylation of MASTL and EEF2K were associated with TP53 mutations, independent of the cancer subtype.

Beyond the examination of full proteomes, the technology also enables analysis of subproteomes, such as specific cell organelles and protein interactions. One of the recent clinically attractive applications is in the field of immuneoncology. Immunopeptidomics is an emerging field in proteomics, in which major histocompatibility complex molecules are isolated, followed by MS analysis of the bound peptides that are presented to the immune system. This approach reveals all of the antigens expressed by the cancer cells and can serve as a platform for neo-antigen identification toward individualized cancer vaccine development. ${ }^{26-30}$ Altogether, analysis of the proteomic level can be integrated with genomics and can complement it by revealing the functional outputs of genomics, integrating with environmental cues that affect the phenotype.

\section{Breast Cancer Tissue Analysis}

Proteomic analysis of cell lines has become a routine procedure that enables coverage of near complete proteomes. Analysis of tumor tissue samples, however, requires various adaptations to reach similar depth. The main challenge is associated with the heterogeneous composition of the tissue, which includes multiple cell types and extracellular matrix (ECM) proteins. Highly abundant ECM proteins may mask the more lowly expressed cellular proteins and may lead to reduced proteome coverage. In addition, analysis of a mixed cell population, which includes cancer cells, together with immune cells, fibroblasts, adipocytes, and endothelial cells, may average out substantial differences associated with each of these populations. To obtain accurate, clinically relevant data, it is therefore essential to dissect the tissue and to extract the specific cells of interest. Tissue macrodissection or laser capture microdissection, isolates the cancer cells from the rest of the tissue, thereby allowing more accurate cancer cell analysis. Microdissected tissue analysis has clear biological advantages and showed higher peptide identification rates because of the lower complexity of the tissue. ${ }^{31}$ However, analysis of minute tissue amounts requires substantial optimization of protein extraction and digestion. ${ }^{32}$ An additional challenge in the analysis of clinical samples is the ability to use archived formalin-fixed, paraffin-embedded tissue. Reversal of the formalin crosslinks is routine in antigen retrieval procedure in 
immunohistochemistry methods. In a similar manner, sample boiling in high detergent concentrations denatures the proteins in the samples and allows exposure of the entire protein to the proteolytic enzymes and results in high digestion efficiency. Comparison of fresh-frozen tissue to formalin-fixed, paraffin-embedded tissue digestion showed that fixation does not reduce the number of identified proteins and does not induce specific crosslinking-related modifications. ${ }^{33,34}$ Therefore, for proteomic analysis there is no actual limitation in the types of analyzed tissues.

Because of the inability to amplify proteins (as opposed to nucleic acids), sample amounts are often limiting the depth of the identified proteome. To overcome this challenge, several approaches have been developed aiming to minimize sample handling and sample loss, by using a single-reactor for the entire sample preparation procedure. The in StageTip sample-processing method uses pipette tips with various filter for protein digestion, separation, and purification. ${ }^{35}$ The solid phase-enhanced sample preparation platform uses paramagnetic beads to perform the protein digestion and purification in a single tube. This method demonstrated improved efficiency and reduced sample loss. ${ }^{36}$ The solid phase-enhanced sample preparation-clinical tissue proteomics platform can be easily adapted to high-throughput format for various clinical samples. ${ }^{37}$

Dozens of proteomic studies analyzed clinical breast tumor samples; however, most suffer from low proteome coverage or cohort size. Rezaul et $\mathrm{al}^{38}$ studied protein expression profiles associated with estrogen receptor (ER) status of breast cancer and obtained a signature of 236 differentially expressed proteins between ER-positive and -negative tumors. Among those, they found Iprin- $\alpha 1$, Fascin, death-associated protein 5 , and $\beta$-arrestin 1 as potential biomarkers of the ER-negative subgroup. However, the cohort consisted of only six patients and an average of 1000 identified proteins per sample. Similarly, Gamez-Pozo et $\mathrm{al}^{39}$ reported identification of $>1600$ protein groups in a cohort of five triple-negative breast cancer (TNBC) samples. With the use of a set of 18 matched normal breast epithelial samples and ER-positive malignant breast epithelial samples, Cha et al ${ }^{40}$ obtained a molecular signature that corresponds to the transition of normal epithelial tissue to highly invasive malignant one and identified 298 significantly changing proteins that were more abundant in the tumor samples. These were enriched for biological processes such as focal adhesion and lipid metabolism.

With the improvement of MS technologies and sample preparation protocols, the size of cohorts and the quality of proteomic data significantly improved. Liu et $\mathrm{al}^{41}$ analyzed a cohort of 126 TNBC breast cancer samples using laser capture microdissection-liquid chromatography-MS/MS approach. The total protein coverage obtained was $>3500$ proteins, and they identified an 11-protein signature for TNBC with 10 proteins that were up-regulated $(C M P K 1$, AIFM1, FTH1, EML4, GANAB, CTNNA1, APIG1, STX12,
$A P 1 M 1$, and $C A P Z B)$ ), and one was down-regulated (methylenetetrahydrofolate dehydrogenase 1) in goodprognosis patients. The signature presented high predictive value of patient prognosis with area under the curve of 0.83 of a receiver operating characteristics curve. With the use of the same techniques De Marchi et $\mathrm{al}^{42}$ obtained a fourprotein signature (programmed cell death protein 4, cingulin, ovarian carcinoma immunoreactive antigen domaincontaining protein 1, and Ras GTPase-activating proteinbinding protein 2 ), which predicts tamoxifen-susceptibility in recurrent breast cancer. The cohort consisted of 112 ERpositive tumor samples with total coverage of 4000 proteins. In our laboratory, we profiled ER-positive breast cancer progression, by comparing matched normal noncancerous tissue and primary tumors with and without lymph node involvement. The cohort was composed of four groups with a total of 88 samples, and the analysis reached the depth of $>9000$ quantified proteins. This work demonstrated metabolic remodeling represented by up-regulation of oxidative phosphorylation processes and down-regulation of key glycolytic proteins such as glyceraldehyde-3 phosphate dehydrogenase, fructose-bisphosphate aldolase $\mathrm{A}$, hexokinase2 , and L-lactate dehydrogenase A and B chains in the cancer samples. Interestingly, these results demonstrate the marked metabolic differences between breast cancer subtypes (discussed below). These metabolic changes coincided with elevation of protein turnover rates, as demonstrated by their elevated synthesis and degradation. Finally, to our surprise, only minor differences were found between the primary tumors and the matched lymph node metastases. ${ }^{43}$ Overall, this work demonstrates the importance of proteomic approach for the understanding of cancer development and provides functional insights in the ER-positive breast cancer.

Two recent studies used state-of-the-art proteomics techniques to examine the differences between breast cancer subtypes and compared these with genome-level differences. Mertins et $\mathrm{al}^{18}$ in the Clinical Proteomic Tumor Analysis Consortium breast cancer study performed the first integrated study of proteomics, phosphoproteomics, and genomics of 77 breast cancer samples from the TCGA cohort. They reached a total depth of $>11,000$ proteins and 26,000 phosphorylation sites, which were then compared with the TCGA data to discern the effects of somatic mutations on the proteomic and phosphoproteomic profiles. A comparison with the intrinsic breast cancer subtypes showed that the abundance of ER, progesterone receptor, human epidermal growth factor receptor 2 (HER2), tumor protein p53, phosphatidylinositol-4,5-bisphosphate 3-kinase catalytic subunit $\alpha$, and GATA binding protein 3 agrees with the mRNA intrinsic subtypes, but other proteins/genes presented some discordance with transcriptomics-based classification. In addition, they performed an unsupervised clustering of the samples to examine the ability to identify novel subtypes. On the proteomic level, three main clusters were identified as luminal enriched, basal enriched, and 


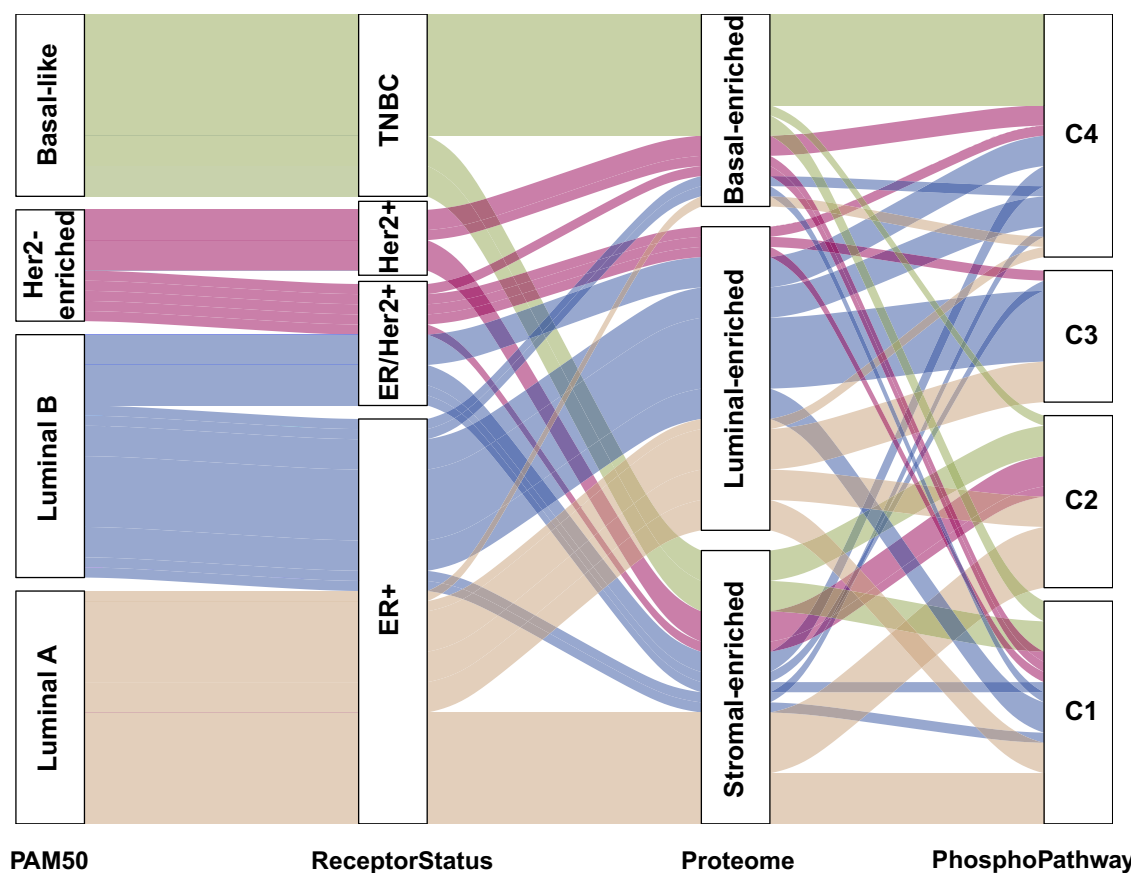

Figure 2 Alluvial plot of 77 breast cancer specimens demonstrating integration of transcriptomic, immunohistochemistry, proteomic, and phosphoproteomic subtypes of breast cancer classification. This image was generated from data originally presented in Mertins et al. ${ }^{18} \mathrm{C} 1$ to $\mathrm{C} 4$ represent phosphoproteomics-based clusters: Cluster 1 (C1) enriched for $G$ protein, $G$ protein -coupled receptor, inositol phosphate metabolism; Cluster 2 (C2) enriched for epidermal growth factor family of receptor tyrosine kinase (ERBB)/human epidermal growth factor receptor (HER), vascular endothelial growth factory (VEGF) signaling, integrin signaling; Cluster 3 (C3) enriched for estrogen receptor (ER) signaling, transcriptional regulation; Cluster 4 (C4) enriched for cell cycle regulation, checkpoint regulation. PAM50, prediction analysis of microarray 50; TNBC, triple-negative breast cancer. stromal enriched. The stromal-enriched cluster consisted of all intrinsic subtypes, potentially dominated by high ECM levels, and the Her2-enriched tumors were distributed among these three clusters, showing the lower relevance of this subtype to the global proteomic profiles (Figure 2). When the phosphoproteomic data were used for pathwaybased classification, four subgroups were identified, including a stromal-enriched group, luminal, basal-enriched, and a mixed group. ${ }^{18}$ Interestingly, overall there was little association between the intrinsic subtypes and the phosphoproteomics-based classification (Figure 2). Nevertheless, cluster 3, which was enriched for ER signaling, did not include any basal tumors, and cluster 4 , which was enriched for cell cycle processes, did not include any luminal A tumors, which are known to be less proliferative.

In a similar study, we have previously analyzed a cohort of 40 samples from ER-positive, triple-negative, and Her2positive tumors and reached the quantitative depth of $>10,000$ proteins. ${ }^{44}$ In agreement with Mertins et al, ${ }^{18}$ we also found only partial concordance with the prediction analysis of microarray $50 \mathrm{mRNA}$-based classifier. This study demonstrated the functional distinction between the classical subtypes, including up-regulation of amino acid, fatty acid oxidation pathways in the ER-positive group, and up-regulation of cell proliferation pathways and higher glycolysis and serine synthesis in TNBC. ${ }^{44}$ These studies reflect the maturation of tissue proteomics in the past years, which enabled it to reach the analytical depth, throughput, and robustness that are necessary to apply it to a broad range of clinical questions. Learning from the mRNA experience, increasing cohort size and tissue microdissection may still dramatically affect protein-level breast cancer classification, but with the accumulation of state-of-the-art studies, it is likely that these protein-based approaches will highlight novel aspects of breast cancer pathogenesis.

\section{Proteomic Analysis of Body Fluids}

Beyond the analysis of tissues, proteomics can be applied to body fluids, for the discovery of cancer biomarkers and for their monitoring in clinical diagnosis. Protein biomarkers are already monitored in routine clinical practice for cancer diagnosis, among them cancer antigen 125, prostate-specific antigen, and carcinoembryonic antigen. However, there is an urgent need to identify additional biomarkers for early diagnosis, to monitor cancer progression, and for companion diagnostics to predict drug response. In recent years, genomic technology expanded its application to biomarker identification through the discovery of mutated DNA in the plasma, as liquid biopsies. ${ }^{45}$ The genomic approaches have the advantage of the high specificity of the mutated DNA. However, often mutations are only recognized in the blood in late stages of tumorigenesis, when the tumors are already invasive. Proteomic approaches, however, may identify proteins secreted by the cancer cells, as well as proteins secreted by the microenvironment in response to the tumorigenic process. Despite the great diagnostic potential, body fluids pose a great challenge in proteomics because of the tremendous dynamic range of protein abundance. Body fluids, primarily serum or plasma, contain large amounts of core plasma proteins, such as albumin, hemoglobin, immunoglobulins, and coagulation factors. The abundance of these proteins is orders of magnitude higher than that of 
tissue leakage proteins, which include the potential biomarkers, ${ }^{46}$ leading to their masking as well as to overall very limited coverage of the plasma proteome. A multitude of methods have been developed to address this challenge. The most commonly used approach is based on immunedepletion of the abundant proteins. ${ }^{47}$ Elimination of albumin, immunoglobulins, apolipoproteins, coagulation factors, and so forth partially overcomes their masking effect and allows identification of hundreds of plasma proteins. ${ }^{47,48} \mathrm{~A}$ complementary approach enriches low abundant proteins through binding to ProteoMiner beads (Bio-Rad Laboratories, Inc., Hercules, CA), which are coated with a combinatorial ligand library composed by millions of hexapeptides that offer noncovalent binding sites to capture proteins. ${ }^{49}$ In this way, the beads allow equal representation of high and low abundant plasma proteins. Suman et $\mathrm{al}^{50}$ applied this approach to breast cancer and analyzed pooled breast cancer subtypes samples. They identified altogether 163 proteins and extracted four subtype markers. Because these approaches were still found to be insufficient to reach the necessary depth for identification of potential cancer biomarkers, alternative techniques fractionate the sample either biochemically or biologically, aiming to reach higher coverage. Biochemical fractionation separates the peptides or proteins to numerous fractions, thereby reducing sample complexity in each fraction. With the use of this approach Keshishian et $\mathrm{al}^{51}$ recently reached the highest coverage of plasma proteins of 5300 proteins by reversephase fractionation into 30 fractions. For the first time, analysis of the plasma provided a comprehensive view of the plasma proteome, but this approach dramatically compromised the throughput. Application of similar approaches to breast cancer plasma samples identified candidate biomarkers of breast cancer subtypes ${ }^{52}$ and markers of early breast cancer diagnosis ${ }^{53}$; however, these studies achieved depth of only a few hundred proteins and are still considered preliminary and require much larger patient cohorts to be established as bona fide breast cancer biomarkers. To improve analytical depth, Zeng et $\mathrm{al}^{54}$ developed a method that combined immune depletion of albumin and $\mathrm{IgG}$, extensive fractionation by isoelectric focusing, and glycoprotein enrichment by multilectin enrichment followed by trypsin digest. Instead of multiple lectins, periodate oxidation-hydrazide chemistry method was developed to enrich the glycoproteome. ${ }^{55,56}$ Although these approaches have the potential to reach comprehensive proteome coverage, they suffer from extensive sample preparation and very low throughput.

To increase analytical throughput, sample preparation developments aimed to reach rapid sample preparation procedures, no fractionation, and combined with optimal chromatographic and MS conditions. Recently, Geyer et al ${ }^{57}$ achieved similar plasma proteome coverage even without immune depletion procedures or fractionation. With the use of these techniques, the obtained coverage was still considerably low and insufficient for cancer states. ${ }^{58} \mathrm{~A}$ distinct approach is based on biological fractionation that isolates microvesicles from the plasma. Because these are largely devoid of the core plasma proteins, it provides high coverage and high throughput. Extracellular vesicles are shed from cells all over the body and can be released into the bloodstream. ${ }^{59}$ There are three main types of extracellular vesicles, which differ in their size, content, and secretion mechanism. ${ }^{60}$ Exosomes (50 to $100 \mathrm{~nm}$ ) are secreted through the multivesicular body system, microparticles (100 to $1000 \mathrm{~nm}$ ) are shed from plasma membrane invaginations, and apoptotic bodies ( 1 to $4 \mu \mathrm{m}$ ) are formed during apoptotic cell death and often contain organelles. We have recently published a deep plasma microparticle proteomics study in which we identified $>3000$ proteins in single MS runs with high-throughput sample preparation. ${ }^{61}$ Although this approach is highly promising for discovery of biomarkers, its major limitation is that the biomarker concentrations differ from those in the unfractionated plasma; therefore, to apply these techniques to routine clinical use the reproducibility and accuracy of microparticle isolation still require improvement and standardization. Altogether, these studies form the technological basis for future biomarker discovery studies. With the accumulation of many of these studies and identification of bona fide biomarkers, these will be able to be examined in the clinic in routine diagnostic test.

\section{Application to the Clinic}

The next step in clinical proteomics is to apply MS-based protein measurements for diagnosis in the clinic. Proteins are routinely measured in the clinic using antibody-based techniques, primarily immunohistochemistry (IHC) and enzyme-linked immunosorbent assays (ELISAs). ${ }^{62,63}$ Although antibodies provide the necessary sensitivity and throughput for routine clinical use, these approaches are challenged by requirement for very highly specific antibodies, and the fact that immunoreactivity can be compromised because of post-translational modifications of the proteins. For example, a comparison between IHC and MSbased analysis of the checkpoint proteins programmed death ligand 1 (PDL1) and programmed death ligand 2 (PDL2) showed that PDL1 staining is reduced when the protein is glycosylated. Therefore, lack of PDL1 staining does not necessarily reflect lack of protein expression and activity. In addition, MS-based absolute quantification of these proteins showed similar expression levels, whereas PDL2 could hardly be detected by IHC in most samples. ${ }^{64}$ Despite these challenges, ELISA remains one of the most established techniques in biomarker measurement in the clinic, because of its high throughput, high sensitivity, and quantitative accuracy. ${ }^{65,66}$ However, the development of new ELISAs is expensive because of a requirement of highly specific antibodies and the need to overcome interferences from autoantibodies and contaminants. ${ }^{67}$ Therefore, it takes 
approximately 2 years to establish each assay. ${ }^{68}$ MS-based analyses open new possibilities in clinical diagnosis because of their high specificity, multiplexing potential, and low cost. Table 1 summarizes the main characteristics of different protein-based approaches (shotgun, targeted, ELISA, IHC) and the suitability of these technologies for clinical practice and identification/quantification of proteins. Shotgun approach is highly applicative for global protein identification, and in cases where high specificity, multiplexing, and rapid configuration are required. However, such a discovery approach is less likely to be integrated into clinical practice, because of the limited reproducibility, and the high level of specialization that is required for measurement and data analysis. The targeted approach is highly suitable when medium-high sensitivity, high specificity, and easy multiplexing are required. ELISA is highly appropriate for clinical tests that require high throughput and sensitivity. IHC is well established in clinical routine and is currently the top approach in providing spatial information. Thus, MS-based approaches meet the requirements of current technologies and potentially can overcome some of their limitations. ${ }^{69}$

To transform the MS techniques described above into clinically relevant platforms they need to provide the throughput and quantitative accuracy to determine the levels of biomarkers in a timely manner. ${ }^{70}$ First, one has to overcome preanalytical and analytical variability in sample preparation and MS analysis. In a similar manner to all clinical tests, improper sample handling and variation in tissue processing protocols and plasma and serum handling will dramatically affect MS-based assays. To that end, simple, rapid, and automated assays are being developed. For example, single reactor approaches for sample preparation dramatically reduce variation in sample preparation. ${ }^{57}$ Targeted assays with heavy standards (described below) further minimize the variability of the MS analyses themselves.

The most attractive approach that was already successfully integrated into clinical practice is the multiple reaction monitoring (MRM) or selected reaction monitoring of targeted MS analysis. The MRM approach overcomes many of the limitations of ELISA and provides the required specificity, multiplexing, and quantification accuracy. Assays can be rapidly configured and do not depend on the development of a high-specificity immunoaffinity reagent. ${ }^{10}$ As opposed to the nontargeted approach described above, MRM analysis is based on rapid selection and fragmentation of proteotypic peptides and monitoring of three to five predefined fragment ions. To overcome the challenge of the identification of optimal proteotypic peptides, which provide unique protein mapping, large efforts were invested to create databases such as the PeptideAtlas. ${ }^{71,72}$ It is a publicly available multiorganism repository of peptides identified in a large set of tandem MS proteomics experiments that allows translation data from an exploratory field to robust quantification. ${ }^{73,74}$ Monitoring of only a limited number of preselected ions allows highly sensitive detection of nanogram per milliliter concentrations of peptides in biological samples'; however, sensitivity cannot yet compete with immunoassays for hormonal and cytokine blood markers. To overcome the sensitivity limitation, MRM assays have been combined with immune assays to enrich for low abundant peptides. Such combination is known as Stable Isotope Standards and Capture by AntiPeptide Antibodies (SISCAPA). It uses peptide-specific antibodies to enrich the native peptides of interest and spiked stable isotope-labeled peptides, which serve as standards for absolute quantification. ${ }^{75,76}$ These peptides are then analyzed by MRM assays to provide the high specificity and quantitative information. SISCAPA allows higher sensitivity because of the antibody enrichment step; however, antibody development makes the assay development more complex and the assay itself more expensive. Of importance, the antibodies do not have to be as specific as for ELISA because the specificity is gained in the MS. ${ }^{76}$ These SISCAPA and MRM assays can be easily multiplexed and monitor dozens of analytes per assay; therefore, they have the capability to analyze multiple biomarkers in single assays. ${ }^{77-80}$

One of the major limitations of MRM is linked to the low resolving power of the triple-quadrupole MS instruments, in which they are performed. As a result, interferences of target analytes with isobaric background contaminants may lead to mis-identification and quantification. To overcome this

Table 1 Evaluation of Shotgun Proteomics, Targeted Proteomics, ELISA, and IHC Approaches for Their Potential in Clinical Practice

\begin{tabular}{lllll}
\hline Parameters & Shotgun approach & Targeted approach & ELISA & IHC \\
\hline High sensitivity & + & +++ & +++ & +++ \\
High specificity & +++ & +++ & ++ & ++ \\
Easy multiplexing & +++ & +++ & + & + \\
Rapid configuration & +++ & ++ & + & + \\
High throughput & + & ++ & + & ++ \\
Spatial information & + & + & + & +++ \\
Global protein identification & +++ & ++ & +++ & + \\
Established in clinics & + & & ++ & ++ \\
\hline
\end{tabular}

+ , limited suitability; ++ , suitable; +++ , highly suitable; ELISA, enzyme-linked immunosorbent assay; IHC, immunohistochemistry. 
limitation it is necessary to examine multiple ions from each protein of interest, thereby limiting the multiplexing capabilities. An alternative approach is the parallel reaction monitoring approach, which provides targeted analysis on high resolution mass spectrometers. ${ }^{81,82}$

\section{Future Perspectives}

Proteomics, as an emerging technology, makes its first steps into the clinical world. Although it has not yet made a large clinical impact, based on its large potential, major efforts should be invested to leap into the next steps of routine clinical use and toward integration into clinical research, aiming to reveal the proteomic layer, complimentary to the genomic ones. These efforts should join clinicians, proteomics experts, and computational biologists, each one contributing its expertise. The technology will undoubtedly further develop in the coming years, enabling better proteome coverage and higher throughput; however, already today major discoveries can be made with current state-of-the-art techniques. Similar to the functional genomics studies, which expanded from panels of 70 tumors to thousands of samples, with current multiplexing capabilities and speed of MS analysis, reaching these aims is within reach. Furthermore, adding the spatial information of the tumor into the analysis will ultimately link histopathologic information with global protein profiles. Such integrated analyses may reveal new regulatory mechanisms underlying tumor heterogeneity and drug resistance, and highlighting potential new therapeutic targets.

Moving the MS technology beyond discovery into clinical diagnostics is presumably a larger challenge; however, the great potential benefits are even more dramatic. We envision that in the future, routine blood tests will be analyzed with multiplexed targeted assays that measure dozens of biomarkers, with high throughput and low cost. Thus, rather than examining only a limited subset of proteins, multiple biomarkers will be monitored routinely, potentially enabling cancer diagnosis at an early stage. Altogether, with the maturation of all of these technologies, multicenter, interdisciplinary collaborations can move proteomics into the clinic to provide better diagnosis and treatment.

\section{References}

1. Aebersold R, Mann M: Mass-spectrometric exploration of proteome structure and function. Nature 2016, 537:347-355

2. Mann M, Kulak NA, Nagaraj N, Cox J: The coming age of complete, accurate, and ubiquitous proteomes. Mol Cell 2013, 49:583-590

3. Bantscheff M, Lemeer S, Savitski MM, Kuster B: Quantitative mass spectrometry in proteomics: critical review update from 2007 to the present. Anal Bioanal Chem 2012, 404:939-965

4. Nesvizhskii AI: A survey of computational methods and error rate estimation procedures for peptide and protein identification in shotgun proteomics. J Proteomics 2010, 73:2092-2123
5. Richards AL, Merrill AE, Coon JJ: Proteome sequencing goes deep Curr Opin Chem Biol 2015, 24:11-17

6. Scheltema RA, Hauschild JP, Lange O, Hornburg D, Denisov E, Damoc E, Kuehn A, Makarov A, Mann M: The Q Exactive HF, a Benchtop mass spectrometer with a pre-filter, high-performance quadrupole and an ultra-high-field Orbitrap analyzer. Mol Cell Proteomics 2014, 13:3698-3708

7. Beck M, Schmidt A, Malmstroem J, Claassen M, Ori A, Szymborska A, Herzog F, Rinner O, Ellenberg J, Aebersold R: The quantitative proteome of a human cell line. Mol Syst Biol 2011, $7: 549$

8. Nagaraj N, Wisniewski JR, Geiger T, Cox J, Kircher M, Kelso J, Paabo S, Mann M: Deep proteome and transcriptome mapping of a human cancer cell line. Mol Syst Biol 2011, 7:548

9. Gillette MA, Carr SA: Quantitative analysis of peptides and proteins in biomedicine by targeted mass spectrometry. Nat Methods 2013 , 10:28-34

10. Picotti P, Aebersold R: Selected reaction monitoring-based proteomics: workflows, potential, pitfalls and future directions. Nat Methods 2012, 9:555-566

11. Kriegsmann J, Kriegsmann M, Casadonte R: MALDI TOF imaging mass spectrometry in clinical pathology: a valuable tool for cancer diagnostics. Int J Oncol 2015, 46:893-906

12. Akbani R, Ng PK, Werner HM, Shahmoradgoli M, Zhang F, Ju Z, Liu W, Yang JY, Yoshihara K, Li J, Ling S, Seviour EG, Ram PT, Minna JD, Diao L, Tong P, Heymach JV, Hill SM, Dondelinger F, Stadler N, Byers LA, Meric-Bernstam F, Weinstein JN, Broom BM, Verhaak RG, Liang H, Mukherjee S, Lu Y, Mills GB: A pan-cancer proteomic perspective on The Cancer Genome Atlas. Nat Commun 2014, 5:3887

13. Uhlen M, Fagerberg L, Hallstrom BM, Lindskog C, Oksvold P, Mardinoglu A, et al: Proteomics. Tissue-based map of the human proteome. Science 2015, 347:1260419

14. Cancer Genome Atlas Network: Comprehensive molecular portraits of human breast tumours. Nature 2012, 490:61-70

15. Nik-Zainal S, Davies H, Staaf J, Ramakrishna M, Glodzik D, Zou X, et al: Landscape of somatic mutations in 560 breast cancer wholegenome sequences. Nature 2016, 534:47-54

16. Curtis C, Shah SP, Chin SF, Turashvili G, Rueda OM, Dunning MJ, Speed D, Lynch AG, Samarajiwa S, Yuan Y, Graf S, Ha G, Haffari G, Bashashati A, Russell R, McKinney S; METABRIC Group, Langerod A, Green A, Provenzano E, Wishart G, Pinder S, Watson P, Markowetz F, Murphy L, Ellis I, Purushotham A, Borresen-Dale AL, Brenton JD, Tavare S, Caldas C, Aparicio S: The genomic and transcriptomic architecture of 2,000 breast tumours reveals novel subgroups. Nature 2012, 486:346-352

17. Geiger T, Cox J, Mann M: Proteomic changes resulting from gene copy number variations in cancer cells. PLoS Genet 2010, 6:e1001090

18. Mertins P, Mani DR, Ruggles KV, Gillette MA, Clauser KR, Wang P, et al: Proteogenomics connects somatic mutations to signalling in breast cancer. Nature 2016, 534:55-62

19. Aviner R, Shenoy A, Elroy-Stein O, Geiger T: Uncovering hidden layers of cell cycle regulation through integrative multi-omic analysis. PLoS Genet 2015, 11:e1005554

20. Jovanovic M, Rooney MS, Mertins P, Przybylski D, Chevrier N Satija R, Rodriguez EH, Fields AP, Schwartz S, Raychowdhury R, Mumbach MR, Eisenhaure T, Rabani M, Gennert D, Lu D, Delorey T, Weissman JS, Carr SA, Hacohen N, Regev A: Immunogenetics. Dynamic profiling of the protein life cycle in response to pathogens. Science 2015, 347:1259038

21. Schwanhausser B, Busse D, Li N, Dittmar G, Schuchhardt J, Wolf J, Chen W, Selbach M: Global quantification of mammalian gene expression control. Nature 2011, 473:337-342

22. Li J, Duncan DT, Zhang B: CanProVar: a human cancer proteome variation database. Hum Mutat 2010, 31:219-228

23. Zhang M, Wang B, Xu J, Wang X, Xie L, Zhang B, Li Y, Li J: CanProVar 2.0: an updated database of human cancer proteome variation. J Proteome Res 2017, 16:421-432 
24. Yang X, Lazar IM: XMAn: a Homo sapiens mutated-peptide database for the MS analysis of cancerous cell states. J Proteome Res 2014, 13: $5486-5495$

25. Lawrence RT, Perez EM, Hernandez D, Miller CP, Haas KM, Irie HY, Lee SI, Blau CA, Villen J: The proteomic landscape of triple-negative breast cancer. Cell Rep 2015, 11:630-644

26. Bassani-Sternberg M, Barnea E, Beer I, Avivi I, Katz T, Admon A: Soluble plasma HLA peptidome as a potential source for cancer biomarkers. Proc Natl Acad Sci U S A 2010, 107:18769-18776

27. Bassani-Sternberg M, Braunlein E, Klar R, Engleitner T, Sinitcyn P, Audehm S, Straub M, Weber J, Slotta-Huspenina J, Specht K, Martignoni ME, Werner A, Hein R, H Busch D, Peschel C, Rad R, Cox J, Mann M, Krackhardt AM: Direct identification of clinically relevant neoepitopes presented on native human melanoma tissue by mass spectrometry. Nat Commun 2016, 7:13404

28. Bassani-Sternberg M, Coukos G: Mass spectrometry-based antigen discovery for cancer immunotherapy. Curr Opin Immunol 2016, 41: $9-17$

29. Hickman HD, Yewdell JW: Mining the plasma immunopeptidome for cancer peptides as biomarkers and beyond. Proc Natl Acad Sci U S A 2010, 107:18747-18748

30. Kalaora S, Barnea E, Merhavi-Shoham E, Qutob N, Teer JK, Shimony N, Schachter J, Rosenberg SA, Besser MJ, Admon A, Samuels Y: Use of HLA peptidomics and whole exome sequencing to identify human immunogenic neo-antigens. Oncotarget 2016, 7: $5110-5117$

31. De Marchi T, Braakman RB, Stingl C, van Duijn MM, Smid M, Foekens JA, Luider TM, Martens JW, Umar A: The advantage of laser-capture microdissection over whole tissue analysis in proteomic profiling studies. Proteomics 2016, 16:1474-1485

32. Braakman RB, Tilanus-Linthorst MM, Liu NQ, Stingl C, Dekker LJ, Luider TM, Martens JW, Foekens JA, Umar A: Optimized nLC-MS workflow for laser capture microdissected breast cancer tissue. J Proteomics 2012, 75:2844-2854

33. Sprung RW Jr, Brock JW, Tanksley JP, Li M, Washington MK, Slebos RJ, Liebler DC: Equivalence of protein inventories obtained from formalin-fixed paraffin-embedded and frozen tissue in multidimensional liquid chromatography-tandem mass spectrometry shotgun proteomic analysis. Mol Cell Proteomics 2009, 8: 1988-1998

34. Ostasiewicz P, Zielinska DF, Mann M, Wisniewski JR: Proteome, phosphoproteome, and N-glycoproteome are quantitatively preserved in formalin-fixed paraffin-embedded tissue and analyzable by high-resolution mass spectrometry. J Proteome Res 2010, 9: 3688-3700

35. Kulak NA, Pichler G, Paron I, Nagaraj N, Mann M: Minimal, encapsulated proteomic-sample processing applied to copy-number estimation in eukaryotic cells. Nat Methods 2014, 11:319-324

36. Hughes CS, Foehr S, Garfield DA, Furlong EE, Steinmetz LM, Krijgsveld J: Ultrasensitive proteome analysis using paramagnetic bead technology. Mol Syst Biol 2014, 10:757

37. Hughes CS, McConechy MK, Cochrane DR, Nazeran T, Karnezis AN, Huntsman DG, Morin GB: Quantitative profiling of single formalin fixed tumour sections: proteomics for translational research. Sci Rep 2016, 6:34949

38. Rezaul K, Thumar JK, Lundgren DH, Eng JK, Claffey KP, Wilson L, Han DK: Differential protein expression profiles in estrogen receptorpositive and -negative breast cancer tissues using label-free quantitative proteomics. Genes Cancer 2010, 1:251-271

39. Gamez-Pozo A, Ferrer NI, Ciruelos E, Lopez-Vacas R, Martinez FG, Espinosa E, Vara JA: Shotgun proteomics of archival triple-negative breast cancer samples. Proteomics Clin Appl 2013, 7:283-291

40. Cha S, Imielinski MB, Rejtar T, Richardson EA, Thakur D, Sgroi DC, Karger BL: In situ proteomic analysis of human breast cancer epithelial cells using laser capture microdissection: annotation by protein set enrichment analysis and gene ontology. Mol Cell Proteomics 2010, 9:2529-2544
41. Liu NQ, Stingl C, Look MP, Smid M, Braakman RB, De Marchi T, Sieuwerts AM, Span PN, Sweep FC, Linderholm BK, Mangia A, Paradiso A, Dirix LY, Van Laere SJ, Luider TM, Martens JW, Foekens JA, Umar A: Comparative proteome analysis revealing an 11-protein signature for aggressive triple-negative breast cancer. J Natl Cancer Inst 2014, 106:djt376

42. De Marchi T, Liu NQ, Stingl C, Timmermans MA, Smid M, Look MP, Tjoa M, Braakman RB, Opdam M, Linn SC, Sweep FC, Span PN, Kliffen M, Luider TM, Foekens JA, Martens JW, Umar A: 4-protein signature predicting tamoxifen treatment outcome in recurrent breast cancer. Mol Oncol 2016, 10:24-39

43. Pozniak Y, Balint-Lahat N, Rudolph JD, Lindskog C, Katzir R, Avivi C, Ponten F, Ruppin E, Barshack I, Geiger T: System-wide clinical proteomics of breast cancer reveals global remodeling of tissue homeostasis. Cell Syst 2016, 2:172-184

44. Tyanova S, Albrechtsen R, Kronqvist P, Cox J, Mann M, Geiger T: Proteomic maps of breast cancer subtypes. Nat Commun 2016, 7: 10259

45. Crowley E, Di Nicolantonio F, Loupakis F, Bardelli A: Liquid biopsy: monitoring cancer-genetics in the blood. Nat Rev Clin Oncol 2013, 10:472-484

46. Jacobs JM, Adkins JN, Qian WJ, Liu T, Shen Y, Camp DG 2nd, Smith RD: Utilizing human blood plasma for proteomic biomarker discovery. J Proteome Res 2005, 4:1073-1085

47. Tu C, Rudnick PA, Martinez MY, Cheek KL, Stein SE, Slebos RJ, Liebler DC: Depletion of abundant plasma proteins and limitations of plasma proteomics. J Proteome Res 2010, 9:4982-4991

48. Poersch A, Grassi ML, Carvalho VP, Lanfredi GP, Palma Cde S, Greene LJ, de Sousa CB, Carrara HH, Candido Dos Reis FJ, Faca VM: A proteomic signature of ovarian cancer tumor fluid identified by highthroughput and verified by targeted proteomics. J Proteomics 2016, 145:226-236

49. Hartwig S, Czibere A, Kotzka J, Passlack W, Haas R, Eckel J, Lehr S: Combinatorial hexapeptide ligand libraries (ProteoMiner): an innovative fractionation tool for differential quantitative clinical proteomics. Arch Physiol Biochem 2009, 115:155-160

50. Suman S, Basak T, Gupta P, Mishra S, Kumar V, Sengupta S, Shukla Y: Quantitative proteomics revealed novel proteins associated with molecular subtypes of breast cancer. J Proteomics 2016, 148:183-193

51. Keshishian H, Burgess MW, Gillette MA, Mertins P, Clauser KR, Mani DR, Kuhn EW, Farrell LA, Gerszten RE, Carr SA: Multiplexed, quantitative workflow for sensitive biomarker discovery in plasma yields novel candidates for early myocardial injury. Mol Cell Proteomics 2015, 14:2375-2393

52. Correa S, Panis C, Binato R, Herrera AC, Pizzatti L, Abdelhay E: Identifying potential markers in breast cancer subtypes using plasma label-free proteomics. J Proteomics 2017, 151:33-42

53. Amon LM, Pitteri SJ, Li CI, McIntosh M, Ladd JJ, Disis M, Porter P, Wong CH, Zhang Q, Lampe P, Prentice RL, Hanash SM: Concordant release of glycolysis proteins into the plasma preceding a diagnosis of ER+ breast cancer. Cancer Res 2012, 72:1935-1942

54. Zeng Z, Hincapie M, Pitteri SJ, Hanash S, Schalkwijk J, Hogan JM, Wang H, Hancock WS: A proteomics platform combining depletion, multi-lectin affinity chromatography (M-LAC), and isoelectric focusing to study the breast cancer proteome. Anal Chem 2011, 83: 4845-4854

55. Yen TY, Macher BA, McDonald CA, Alleyne-Chin C, Timpe LC: Glycoprotein profiles of human breast cells demonstrate a clear clustering of normal/benign versus malignant cell lines and basal versus luminal cell lines. J Proteome Res 2012, 11:656-667

56. Whelan SA, Lu M, He J, Yan W, Saxton RE, Faull KF, Whitelegge JP, Chang HR: Mass spectrometry (LC-MS/MS) sitemapping of $\mathrm{N}$-glycosylated membrane proteins for breast cancer biomarkers. J Proteome Res 2009, 8:4151-4160

57. Geyer PE, Kulak NA, Pichler G, Holdt LM, Teupser D, Mann M: Plasma proteome profiling to assess human health and disease. Cell Syst 2016, 2:185-195 
58. Geyer PE, Wewer Albrechtsen NJ, Tyanova S, Grassl N, Iepsen EW, Lundgren J, Madsbad S, Holst JJ, Torekov SS, Mann M: Proteomics reveals the effects of sustained weight loss on the human plasma proteome. Mol Syst Biol 2016, 12:901

59. D'Souza-Schorey C, Di Vizio D: Biology and proteomics of extracellular vesicles: harnessing their clinical potential. Expert Rev Proteomics 2014, 11:251-253

60. Raposo G, Stoorvogel W: Extracellular vesicles: exosomes, microvesicles, and friends. J Cell Biol 2013, 200:373-383

61. Harel M, Oren-Giladi P, Kaidar-Person O, Shaked Y, Geiger T: Proteomics of microparticles with SILAC Quantification (PROMISQuan): a novel proteomic method for plasma biomarker quantification. Mol Cell Proteomics 2015, 14:1127-1136

62. Lim MJ, Foster GJ, Gite S, Ostendorff HP, Narod S, Rothschild KJ: An ELISA-based high throughput protein truncation test for inherited breast cancer. Breast Cancer Res 2010, 12:R78

63. Tchou J, Lam L, Li YR, Edwards C, Ky B, Zhang H: Monitoring serum HER2 levels in breast cancer patients. Springerplus 2015, 4:237

64. Morales-Betanzos CA, Lee H, Gonzalez-Ericsson PI, Balko JM, Johnson DB, Zimmerman LJ, Liebler DC: Quantitative mass spectrometry analysis of PD-L1 protein expression, N-glycosylation and expression stoichiometry with PD-1 and PD-L2 in human melanoma. Mol Cell Proteomics 2017, [Epub ahead of print] doi:10.1074/mcp. RA117.000037

65. Krastins B, Prakash A, Sarracino DA, Nedelkov D, Niederkofler EE, Kiernan UA, Nelson R, Vogelsang MS, Vadali G, Garces A, Sutton JN, Peterman S, Byram G, Darbouret B, Perusse JR, Seidah NG, Coulombe B, Gobom J, Portelius E, Pannee J, Blennow K, Kulasingam V, Couchman L, Moniz C, Lopez MF: Rapid development of sensitive, high-throughput, quantitative and highly selective mass spectrometric targeted immunoassays for clinically important proteins in human plasma and serum. Clin Biochem 2013, 46:399-410

66. Sapin R: [Interferences in immunoassays: mechanisms and outcomes in endocrinology] French. Ann Endocrinol (Paris) 2008, 69:415-425

67. Wang P, Whiteaker JR, Paulovich AG: The evolving role of mass spectrometry in cancer biomarker discovery. Cancer Biol Ther 2009, 8:1083-1094

68. Tighe PJ, Ryder RR, Todd I, Fairclough LC: ELISA in the multiplex era: potentials and pitfalls. Proteomics Clin Appl 2015, 9:406-422

69. Solier C, Langen H: Antibody-based proteomics and biomarker research - current status and limitations. Proteomics 2014, 14:774-783

70. Grebe SK, Singh RJ: LC-MS/MS in the clinical laboratory - where to from here? Clin Biochem Rev 2011, 32:5-31

71. Kusebauch U, Campbell DS, Deutsch EW, Chu CS, Spicer DA, Brusniak MY, Slagel J, Sun Z, Stevens J, Grimes B, Shteynberg D, Hoopmann MR, Blattmann P, Ratushny AV, Rinner O, Picotti P,
Carapito C, Huang CY, Kapousouz M, Lam H, Tran T, Demir E, Aitchison JD, Sander C, Hood L, Aebersold R, Moritz RL: Human SRMAtlas: a resource of targeted assays to quantify the complete human proteome. Cell 2016, 166:766-778

72. Deutsch EW, Lam H, Aebersold R: PeptideAtlas: a resource for target selection for emerging targeted proteomics workflows. EMBO Rep 2008, 9:429-434

73. Desiere F, Deutsch EW, King NL, Nesvizhskii AI, Mallick P, Eng J, Chen S, Eddes J, Loevenich SN, Aebersold R: The PeptideAtlas project. Nucleic Acids Res 2006, 34:D655-D658

74. Lam H, Deutsch EW, Eddes JS, Eng JK, King N, Stein SE Aebersold R: Development and validation of a spectral library searching method for peptide identification from MS/MS. Proteomics 2007, 7:655-667

75. Anderson NL, Jackson A, Smith D, Hardie D, Borchers C, Pearson TW: SISCAPA peptide enrichment on magnetic beads using an in-line bead trap device. Mol Cell Proteomics 2009, 8 : 995-1005

76. Anderson NL, Anderson NG, Haines LR, Hardie DB, Olafson RW, Pearson TW: Mass spectrometric quantitation of peptides and proteins using Stable Isotope Standards and Capture by Anti-Peptide Antibodies (SISCAPA). J Proteome Res 2004, 3:235-244

77. Razavi M, Anderson NL, Yip R, Pope ME, Pearson TW: Multiplexed longitudinal measurement of protein biomarkers in DBS using an automated SISCAPA workflow. Bioanalysis 2016, 8: $1597-1609$

78. Razavi M, Leigh Anderson N, Pope ME, Yip R, Pearson TW: High precision quantification of human plasma proteins using the automated SISCAPA Immuno-MS workflow. N Biotechnol 2016, 33: 494-502

79. Razavi M, Frick LE, LaMarr WA, Pope ME, Miller CA, Anderson NL, Pearson TW: High-throughput SISCAPA quantitation of peptides from human plasma digests by ultrafast, liquid chromatography-free mass spectrometry. J Proteome Res 2012, 11: $5642-5649$

80. van den Broek I, Nouta J, Razavi M, Yip R, Bladergroen MR, Romijn FP, Smit NP, Drews O, Paape R, Suckau D, Deelder AM, van der Burgt YE, Pearson TW, Anderson NL, Cobbaert CM: Quantification of serum apolipoproteins A-I and B-100 in clinical samples using an automated SISCAPA-MALDI-TOF-MS workflow. Methods 2015, 81:74-85

81. Peterson AC, Russell JD, Bailey DJ, Westphall MS, Coon JJ: Parallel reaction monitoring for high resolution and high mass accuracy quantitative, targeted proteomics. Mol Cell Proteomics 2012, 11: $1475-1488$

82. Gallien S, Duriez E, Crone C, Kellmann M, Moehring T, Domon B: Targeted proteomic quantification on quadrupole-orbitrap mass spectrometer. Mol Cell Proteomics 2012, 11:1709-1723 